\title{
SYSTEMS OF EQUATIONS IN AN INFINITY OF UNKNOWNS, WHOSE SOLUTION INVOLVES AN ARBITRARY PARAMETER*
}

\author{
BY \\ I. M. SHEFFER \\ INTRODUCTION
}

This paper gives a discussion of a system of infinitely many linear equations in infinitely many unknowns. The character of the system and of the results differs widely from that of other papers on this subject. It appears that most of the work on such systems is restricted to solutions in Hilbert space: i.e., where $\sum_{i=1}^{\infty} x_{i}^{2}$ converges, $\left(x_{i}\right)$ being a solution. The paper of Walsh $\dagger$ may be cited as an exception. He obtains a unique solution, however.

The present work may be described as follows :

In $\S I$ we start with a system of linear equations which reduces to a system of difference equations yielding an infinity of solutions. These solutions are shown to be solutions of the original system, after suitable restrictions are laid on the coefficients of the system. We thus get a one-parameter family of solutions. Then we turn, in §II, to a more general system of equations, whose solution can be effected by a method of approximations based on the results of $\S I$. There is shown to exist at least a one-parameter family of solutions $\left(x_{i}\right)$ satisfying the inequality $\left|x_{i}\right| \leqq A P^{i}, P<1$.

In the third section we show that all solutions $\left(x_{i}\right)$ which satisfy an inequality $\left|x_{i}\right| \leqq A P^{i}, P<1$, can be obtained by the method of $\S I I$. In $\S I V$ there is discussed the method of solution by assuming a solution in the form of a power series, then formally equating coefficients, and finally justifying the formal processes. This method appears to be less effective than the one used in §II.

The writer takes occasion here to express his appreciation for the many suggestions made to him by Professor G. C. Evans.

* Presented to the Society, May 3, 1924; received by the editors in October, 1925.

$\dagger$ J. L. Walsh, American Journal of Mathematics, vol. 42 (1920), pp. 91-96.

Some time after submitting this paper to these Transactions the writer's attention was called to the following additional literature: 365.

H. von Koch, 5th International Congress of Mathematicians (Cambridge, 1912), vol. 1, pp. 352-

O. Perron, Mathematische Annalen, vol. 34 (1921), pp. 1-15.

A general reference is to F. Riesz Les Systèmes d'Equations Linéaires d̀ une Infinité d'Inconnues. 
I. LINEAR SYSTEMS REDUCIBLE TO SYSTEMS OF DIFFERENCE EQUATIONS

Consider the infinite system of linear equations

$$
x_{i}+\lambda \sum_{j=i+1}^{\infty} x_{j}=c_{i} \quad(i=1,2,3, \cdots)
$$

where $\left|c_{i}\right| \leqq M P^{i}, P<1$.

THEOREM 1. The system (I) has an infinity of solutions for each $\lambda$ in $|\lambda-1|>1$, and a single solution for each $\lambda$ in $|\lambda-1| \leqq 1$.

Proof. On subtracting successive equations we have

$$
\begin{aligned}
& x_{1}+(\lambda-1) x_{2}=c_{1}-c_{2}, \\
& \cdot \cdot \cdot \cdot \cdot \cdot \cdot \cdot \cdot \\
& x_{n}+(\lambda-1) x_{n+1}=c_{n}-c_{n+1},
\end{aligned}
$$

Every solution of (I) satisfies (II). It remains to be seen when a solution of (II) is also a solution of (I).

Set $\lambda-1=\nu$, and suppose $\nu \neq 0$. Choose $x_{1}=x_{1}^{(0)}$ arbitrarily. Then $x_{2}, x_{3}, \cdots$ of (II) are uniquely determined. So there are infinitely many solutions of (II) when $\nu \neq 0$. They are given by

$x_{1}=x_{1}^{(0)}$

$$
x_{2}=\frac{-x_{1}^{(0)}}{\nu}+\frac{c_{1}-c_{2}}{\nu} \text {, }
$$$$
x_{3}=\frac{x_{1}^{(0)}}{\nu^{2}}-\frac{c_{1}-c_{2}}{\nu^{2}}+\frac{c_{2}-c_{3}}{\nu}
$$

(1)

$$
x_{n}=(-1)^{n-1} \frac{x_{1}^{(0)}}{\nu^{n-1}}+(-1)^{n-2} \frac{c_{1}-c_{2}}{\nu^{n-1}}+(-1)^{n-3} \frac{c_{2}-c_{3}}{\nu^{n-2}}+\cdots+\frac{c_{n-1}-c_{n}}{\nu} \text {, }
$$

Therefore

$$
\left|c_{i}\right| \leqq M P^{i}, P<1 \text {. }
$$

$$
\begin{aligned}
\left|x_{n}\right| & \leqq \frac{\left|x_{1}^{(0)}\right|}{|\nu|^{n-1}}+\frac{M P(1+P)}{|\nu|^{n-1}}+\frac{M P^{2}(1+P)}{|\nu|^{n-2}}+\cdots+\frac{M P^{n-1}(1+P)}{|\nu|} \\
& =\frac{\left|x_{1}^{(0)}\right|}{|\nu|^{n-1}}+M(1+P) P^{n}\left[\frac{1}{\left|P_{\nu}\right|^{n-1}}+\frac{1}{\left|P_{\nu}\right|^{n-2}}+\cdots \cdot+\frac{1}{|P \nu|}\right] .
\end{aligned}
$$


Assume temporarily that $P|\nu|>1$. (Observe that then $|\nu|>1$.) Then

The series

$$
\left|x_{n}\right| \leqq \frac{\left|x_{1}^{(0)}\right|}{|\nu|^{n-1}}+\frac{M(1+P)}{|P \nu|-1} P^{n}\left[1-\frac{1}{|P \nu|^{n-1}}\right] .
$$

converges absolutely. For

$$
S_{i}=x_{i}+\lambda \sum_{j=i+1}^{\infty} x_{j}
$$

$$
\begin{aligned}
\left|x_{i}\right|+|\lambda| \sum_{j=i+1}^{\infty}\left|x_{i}\right| \leqq\left|x_{i}\right|+|\lambda|\left\{\frac{\left|x_{1}^{(0)}\right|}{|\nu|^{i}}\right. & \cdot \frac{1}{1-\frac{1}{\left.\right|_{\nu} \mid}+\frac{M(1+P)}{P|\nu|-1} \cdot \frac{P^{i+1}}{1-P}} \\
& \left.-\frac{M P(1+P)}{\left.\left.(P|\nu|-1)\right|_{\nu}\right|^{i}} \cdot \frac{1}{1-\frac{1}{|\nu|}}\right\} .
\end{aligned}
$$

Let

$$
S_{i, n}=x_{i}+\lambda\left(x_{i+1}+x_{i+2}+\cdots \cdot+x_{n}\right) .
$$

On adding the $i$ th to $n$th equations of (II) we have

$$
S_{i, n}+(\lambda-1) x_{n+1}=c_{i}-c_{n+1} .
$$

Now by hypothesis, $\lim _{n \rightarrow \infty} c_{n}=0$. And from (2) $\lim _{n \rightarrow \infty} x_{n}=0$. Hence $S_{i}=c_{i}$. Therefore $\left(x_{i}\right)$ is a solution of $(\mathrm{I})$. We have thus shown that there are an infinity of solutions of (I) for each $\lambda$ such that $P|\nu|>1$. This conclusion holds for every $\lambda$ in $|\nu|>1$. For let $\nu_{0}$ be any value of $\nu$ such that $\left|\nu_{0}\right|>1$. We can always choose $P^{\prime}$ satisfying $1>P^{\prime} \geqq P$ and such that $P^{\prime}\left|\nu_{0}\right|>1$. And obviously $\left|c_{i}\right| \leqq M P^{\prime i}$. We can therefore replace $P$ by $P^{\prime}$, and so obtain an infinity of solutions for $\nu=\nu_{0}$. This proves the first part of the theorem.

Note: In the inequality for $\left|x_{n}\right|$, we must replace $P$ by $P^{\prime}$ whenever we use $P^{\prime}$.

Now assume a solution $x_{i}(\lambda)$ of $(\mathrm{I})$ in the form of a power series :

$$
x_{i}=A_{0 i}+A_{1 i}(\lambda-1)+\cdots+A_{n i}(\lambda-1)^{n}+\cdots \cdot
$$

Substituting into (I) formally and equating coefficients, we obtain the following equations to determine $A_{i j}$ :

$$
\begin{aligned}
& \sum_{j=i}^{\infty} A_{0 j}=c_{i}, \\
& \sum_{j=i}^{\infty} A_{n j}=-\sum_{j=i+1}^{\infty} A_{n-1, j}=c_{i}{ }^{(n)} .
\end{aligned}
$$


$A_{0 i}=c_{i}-c_{i+1}$ is a solution of the first system, as is evident. Therefore

$$
c_{i}^{(1)}=-\sum_{j=i+1}^{\infty} A_{0 j}=-\sum_{j=i+1}^{\infty}\left(c_{j}-c_{j+1}\right)=-c_{i+1} .
$$

Hence a solution of

$$
\sum_{j=i}^{\infty} A_{1 j}=-\sum_{j=i+1}^{\infty} A_{0 j}
$$

is $A_{1 i}=-\left(c_{i+1}-c_{i+2}\right)$. Therefore

$$
\stackrel{(2)}{c_{i}}=-\sum_{j=i+1}^{\infty} A_{1 j}=c_{i+2} .
$$

Therefore $A_{2 i}=c_{i+2}-c_{i+3}$; and so on. In general, a solution of

is

$$
\sum_{j=i}^{\infty} A_{n j}=c_{i}^{(n)}
$$

Therefore

$$
A_{n i}=(-1)^{n}\left(c_{i+n}-c_{i+n+1}\right) \text {. }
$$

that is

$$
\begin{aligned}
x_{i}=\left(c_{i}-c_{i+1}\right)-(\lambda-1) & \left(c_{i+1}-c_{i+2}\right)+\cdots \\
+ & (-1)^{n}(\lambda-1)^{n}\left(c_{i+n}-c_{i+n+1}\right)+\cdots
\end{aligned}
$$

$$
\begin{aligned}
x_{i}= & {\left[c_{i}-c_{i+1}(\lambda-1)+\cdot \cdot+(-1)^{n}(\lambda-1)^{n} c_{i+n}+\cdot \cdot \cdot\right] } \\
& -\left[c_{i+1}-c_{i+2}(\lambda-1)+\cdot \cdot+(-1)^{n}(\lambda-1)^{n} c_{i+n+1}+\cdot \cdot\right]
\end{aligned}
$$

whenever the two series converge. Since $\left|c_{i}\right| \leqq M P^{i}, P<1$, each series does converge for $|\lambda-1|<1 / P$; and

$$
\left|x_{i}\right| \leqq \frac{M P^{i}}{1-P|\nu|}+\frac{M P^{i+1}}{1-P|\nu|}=\frac{M(1+P)}{1-P|\nu|} P^{i} .
$$

Therefore

$$
\left|x_{i}\right|+|\lambda| \sum_{j=i+1}^{\infty}\left|x_{i}\right| \leqq\left|x_{i}\right|+\frac{M(1+P)|\lambda|}{1-P|\nu|} \cdot \frac{P}{1-P} \cdot P^{i} .
$$

Therefore $x_{i}+\lambda \sum_{j=i+1}^{\infty} x_{j}$ converges uniformly in every circle of radius $<1 / P$. It is therefore legitimate to sum by columns. Consequently

$$
x_{i}+\lambda \sum_{j=i+1}^{\infty} x_{j}=c_{i}
$$

That is, the formal solution is a true solution. Denote it by $\bar{x}_{i}$. Set $x_{i}=y_{i}$ $+\bar{x}_{i}$. Then 


$$
y_{i}+\lambda \sum_{j=i+1}^{\infty} y_{j}=0 \text {. }
$$

If $\lambda=1$ we see that the unique solution is $y_{i}=0$. Suppose $\lambda \neq 1$. Then

$$
y_{i}+(\lambda-1) y_{i+1}=0, \quad \text { or } \quad y_{i}=\frac{(-1)^{i-1}}{(\lambda-1)^{i-1}} y_{1} \text {. }
$$

Add the $i$ th to $n$th equations in (6):

$$
y_{i}+\lambda\left(y_{i+1}+\cdots \cdot+y_{n}\right)=-(\lambda-1) y_{n+1} .
$$

Hence a solution $y_{i} \neq 0$ of (6) will be a solution of (5) if and only if $y_{n} \rightarrow 0$ as $n \rightarrow \infty$, i.e., if and only if $|\lambda-1|>1$. Hence for $|\lambda-1| \leqq 1$ the only solution of (5) is $y_{i}=0$, and therefore $\bar{x}_{i}$ is unique. This completes the proof. $x_{1}^{(0)}$.

COROLlaRY. Every solution for $|\lambda-1|>1$ is obtained by giving all values to

Consider now the system

$$
x_{i}+\sum_{j=i+1}^{\infty} \lambda_{j} x_{j}=c_{i} \quad(i=1,2, \ldots .) .
$$

On subtracting successive equations we obtain

$$
x_{i-1}+\left(\lambda_{i}-1\right) x_{i}=c_{i-1}-c_{i} \text {. }
$$

Choose $x_{1}=x_{1}^{(0)}$ arbitrarily. Then every solution of (II) is given by

$$
x_{1}=x_{1}^{(0)}
$$

$$
x_{n}=\frac{(-1)^{n-1} x_{1}^{(0)}}{\nu_{2} \nu_{3} \cdots \nu_{n}}+\frac{(-1)^{n-2}\left(c_{1}-c_{2}\right)}{\nu_{2} \nu_{3} \cdots \nu_{n}}+\cdots+\frac{c_{n-1}-c_{n}}{\nu_{n}},
$$

where $\nu_{i}=\lambda_{i}-1$. Assume that

$$
\begin{aligned}
& \left|c_{n}\right| \leqq M P^{n}, \quad P<1 ; \\
& \left|\nu_{n}\right| \geqq \alpha>\frac{1}{P} \quad(\text { and therefore } \alpha>1) ; \\
& \sum_{j=1}^{\infty}\left|\lambda_{i}\right| P^{i} \text { converges. }
\end{aligned}
$$

Then

$$
\begin{aligned}
\left|x_{n}\right| & \leqq \frac{\left|x_{1}^{(0)}\right|}{\alpha^{n-1}}+M(1+P) P^{n}\left[\frac{1-\left(\frac{1}{\alpha P}\right)^{n-1}}{\alpha P-1}\right] \\
& \leqq \frac{\left|x_{1}^{(0)}\right|}{\alpha^{n-1}}+\frac{M(1+P)}{\alpha P-1}-P^{n} .
\end{aligned}
$$


Therefore

$$
\lim _{n \rightarrow \infty} x_{n}=0
$$

Also

$$
\left|x_{i}\right|+\sum_{j=i+1}^{\infty}\left|\lambda_{j} x_{j}\right| \leqq\left|x_{i}\right|+\sum_{j=i+1}^{\infty}\left|\lambda_{i}\right|\left\{\frac{\left|x_{1}^{(0)}\right|}{\alpha^{j-1}}+\frac{M(1+P)}{\alpha P-1} P^{i}\right\}
$$

which converges, by (2). Add the $i$ th to $n$th equations of (II) :

$$
x_{i}+\lambda_{i+1} x_{i+1}+\cdots \cdot+\lambda_{n} x_{n}=c_{i}-c_{n}+x_{n} .
$$

Therefore

$$
x_{i}+\sum_{j=i+1}^{\infty} \lambda_{j} x_{j}=c_{i}+\lim _{n \rightarrow \infty}\left(x_{n}-c_{n}\right)=c_{i}
$$

Therefore $\left(x_{i}\right)$ is a solution of $(I)$.

Since $x_{1}^{(0)}$ is arbitrary, there are an infinity of solutions. We have thus

Theorem 2. If, in the system (I) $x_{i}+\sum_{j=i+1}^{\infty} \lambda_{j} x_{j}=c_{i}(i=1,2,3, \cdots)$, we have the inequalities $\left|c_{n}\right| \leqq M P^{n}, P<1$, then for each set $\left(\lambda_{i}\right)$ such that $\left|\lambda_{i}-1\right| \geqq \alpha>1 / P$ and $\sum_{j=1}^{\infty}\left|\lambda_{j}\right| P^{j}$ converges, there are an infinity of solutions $\left(x_{i}\right)$.

Corollary. Every such solution of $(\mathrm{I})$ is obtained by giving to $x_{1}^{(0)}$ all values.

Remarks: (1) Observe that for any such solution $\left(x_{i}\right)$ we have $\left|x_{n}\right| \leqq$ $\left[\left|x_{1}^{(0)}\right| / P+M(1+P) /(P \alpha-1)\right] P^{n} ;$ (2) $\sum_{j=1}^{\infty}\left|\lambda_{j}\right| P^{j}$ converges if $\lim _{j=\infty}$ $\left|\lambda_{j+1} / \lambda_{j}\right|<1 / P$, and therefore $\lambda_{j}$ need not be bounded as $j \rightarrow \infty$.

II. METhod OF APPROXIMATIONS APPLIED to A MORE GENERAL SYSTEM

We now consider the more general system

$$
x_{i}+\sum_{j=i+1}^{\infty}\left(\lambda_{j}+b_{i j}\right) x_{j}=c_{i} \quad(i=1,2, \cdots \cdot) .
$$

Assume that

$$
\begin{aligned}
&\left|c_{i}\right| \leqq M P^{i}, \quad P<1, \\
&\left|\lambda_{i}-1\right| \geqq \alpha>\frac{1}{P}, \quad\left|b_{i j}\right| \leqq N, \\
& \sum_{j=1}^{\infty}\left|\lambda_{j}\right| P^{j} \text { converges. }
\end{aligned}
$$

Let $x_{i}^{(0,0)}$ be any solution of $x_{i}+\sum_{j=1+1}^{\infty} \lambda_{j} x_{j}=c_{i}$, and set $x_{i}^{(1)}=x_{i}-x_{i}^{(0,0)}$. By Theorem 2, a solution $x_{i}^{(0,0)}$ exists. Then, formally, 


$$
\begin{aligned}
x_{i}^{(1)} & =\left\{c_{i}-\sum_{j=i+1}^{\infty}\left(\lambda_{j}+b_{i j}\right) x_{j}\right\}-\left\{c_{i}-\sum_{j=i+1}^{\infty} \lambda_{j} x_{j}{ }^{(0,0)}\right\} \\
& =-\sum_{j=i+1}^{\infty}\left(\lambda_{j}+b_{i j}\right)\left(x_{j}-x_{j}{ }^{(0,0)}\right)-\sum_{j=i+1}^{\infty} b_{i j} x_{j}{ }^{(0,0)}
\end{aligned}
$$

i.e.,

where

$$
x_{i}^{(1)}+\sum_{j=i+1}^{\infty}\left(\lambda_{j}+b_{i j}\right) x_{j}^{(1)}=c_{i}^{(1)}
$$

$$
c_{i}^{(1)}=-\sum_{j=i+1}^{\infty} b_{i j} x_{j}^{(0,0)}
$$

Let $x_{i}^{(0,1)}$ be a solution of

$$
x_{i}+\sum_{j=i+1}^{\infty} \lambda_{j} x_{j}=c_{i}^{(1)} .
$$

It will be shown that $c_{1}^{(1)}$ satisfies the inequality required for Theorem 2, and consequently a solution exists. This remark applies also to the later $c_{i}^{(n)}$ s : $c_{i}^{(2)}, c_{i}^{(3)}, \ldots$. Set

Then

$$
x_{i}^{(2)}=x_{i}^{(1)}-x_{i}^{(0,1)}=-\sum_{j=i+1}^{\infty}\left(\lambda_{j}+b_{i j}\right)\left(x_{j}^{(1)}-x_{j}^{(0,1)}\right)-\sum_{j=i+1}^{\infty} b_{i j} x_{j}^{(0,1)} .
$$

where

$$
x_{i}^{(2)}+\sum_{j=i+1}^{\infty}\left(\lambda_{j}+b_{i j}\right) x_{j}^{(2)}=c_{i}^{(2)}
$$

$$
c_{i}^{(2)}=-\sum_{j=i+1}^{\infty} b_{i j} x_{j}^{(0,1)}
$$

and so on. In general, let $x_{i}^{(0, n-1)}$ be a solution of

and set

$$
x_{i}+\sum_{j=i+1}^{\infty} \lambda_{j} x_{j}=c_{i}^{(n-1)}
$$

Then

$$
x_{i}^{(n)}=x_{i}^{(n-1)}-x_{i}^{(0, n-1)} .
$$

where

$$
x_{i}^{(n)}+\sum_{j=i+1}^{\infty}\left(\lambda_{j}+b_{i j}\right) x_{j}^{(n)}=c_{i}^{(n)}
$$

$$
c_{i}^{(n)}=-\sum_{j=i+1}^{\infty} b_{i j} x_{j}^{(0, n-1)}
$$


We need to consider convergence. In solving the system $x_{i}+\sum_{j=i+1}^{\infty} \lambda_{j} x_{i}=$ $c_{i}$, we obtained the inequality

$$
\left|x_{n}\right| \leqq\left[\frac{\left|x_{1}^{(0)}\right|}{P}+\frac{M(1+P)}{P \alpha-1}\right] P^{n} .
$$

In the present case this is

Therefore

$$
\left|x_{n}^{(0,0)}\right| \leqq\left[\frac{\left|x_{1}^{(0,0)}\right|}{P}+\frac{M(1+P)}{P \alpha-1}\right] P^{n} .
$$

$$
\begin{aligned}
\left|c_{i}^{(1)}\right| \leqq \sum_{i=i+1}^{\infty}\left|b_{i j}\right|\left|x_{i}^{(0,0)}\right| & \leqq N\left[\frac{\left|x_{1}^{(0,0)}\right|}{P}+\frac{M(1+P)}{P \alpha-1}\right] \sum_{j=i+1}^{\infty} P^{i} \\
& =N\left[\frac{\left|x_{1}^{(0,0)}\right|}{P}+\frac{M(1+P)}{P \alpha-1}\right] \frac{P}{1-P} \cdot P^{i}
\end{aligned}
$$

Let

$$
\begin{gathered}
Q=\frac{N}{1-P}, \quad R=\frac{P N(1+P)}{(1-P)(P \alpha-1)}, \\
M^{(1)}=Q\left|x_{1}^{(0,0)}\right|+R M .
\end{gathered}
$$

Then

$$
\left|c_{i}^{(1)}\right| \leqq M^{(1)} P^{i} .
$$

Assume $\alpha$ chosen so that $R<1$. This is equivalent to assuming that

$$
\alpha>\frac{N P^{2}+(N-1) P+1}{P(1-P)} .
$$

(Observe that this inequality implies $P \alpha>1$.) In the solution $x_{1}^{(0,1)}$ of $x_{i}+\sum_{j=i+1}^{\infty} \lambda_{j} x_{j}=c_{i}^{(1)}, x_{1}^{(0,1)}$ can be taken arbitrarily (see Theorem 2). Therefore

Therefore

$$
\left|x_{n}^{(0,1)}\right| \leqq\left[\frac{\left|x_{1}^{(0,1)}\right|}{P}+\frac{M^{(1)}(1+P)}{P \alpha-1}\right] P^{n} .
$$

$$
\begin{aligned}
\left|c_{i}^{(2)}\right| \leqq \sum_{i=i+1}^{\infty}\left|b_{i j}\right|\left|x_{i}^{(0,1)}\right| & \leqq N\left[\frac{\left|x_{1}^{(0,1)}\right|}{P}+\frac{M^{(1)}(1+P)}{P \alpha-1}\right] \frac{P}{1-P} P^{i} \\
& =\left[Q\left|x_{1}^{(0,1)}\right|+R M^{(1)}\right] P^{i} \\
& =M^{(2)} P^{i}, \text { say }
\end{aligned}
$$


and so on. It is clear that in general

and

$$
\left|x_{n}^{(0, r)}\right| \leqq\left[\frac{\left|x_{1}^{(0, r)}\right|}{P}+\frac{M^{(r)}(1+P)}{P \alpha-1}\right] P^{n}
$$

$$
\begin{aligned}
\left|c_{i}^{(r+1)}\right| & \leqq \sum_{j=i+1}^{\infty}\left|b_{i j}\right|\left|x_{j}^{(0, r)}\right| \\
& \leqq\left[Q\left|x_{1}^{(0, r)}\right|+R M^{(r)}\right] P^{i}=M^{(r+1)} P^{i}
\end{aligned}
$$

where $x_{1}^{(0, r)}$ is chosen arbitrarily, and $M^{(r)}$ is defined by the relation

$$
\begin{aligned}
M^{(r+1)} & =Q\left|x_{1}^{(0, r)}\right|+R M^{(r)}, \\
M^{(0)} & =M .
\end{aligned}
$$

From this recurrent relation for $M^{(r)}$ we have

$$
\begin{aligned}
M^{(r)}= & Q\left|x_{1}^{(0, r-1)}\right|+Q R\left|x_{1}^{(0, r-2)}\right|+Q R^{2}\left|x_{1}^{(0, r-3)}\right| \\
& +\cdots+Q R^{r-1}\left|x_{1}^{(0,0)}\right|+R^{r} M .
\end{aligned}
$$

The choice of $x_{1}^{(0,0)}, x_{1}^{(0,1)}, \cdots, x_{1}^{(0, r)}, \cdots$ is arbitrary. Now assume them so chosen that

Then

$$
\left|x_{1}^{(0, r)}\right| \leqq \beta T^{r}, \quad R<T<1 .
$$

$$
\begin{aligned}
M^{(r)} & \leqq \beta Q T^{r-1}\left[1+\frac{R}{T}+\frac{R^{2}}{T^{2}}+\cdots+\frac{R^{r-1}}{T^{r-1}}\right]+M R^{r} \\
& =\beta Q T^{r-1}\left[\frac{1-\left(\frac{R}{T}\right)^{r}}{1-\frac{R}{T}}\right]+M R^{r} \\
& \leqq\left[\frac{\beta Q}{1-\frac{R}{T}}+M R\right] T^{r-1}=E T^{r-1}, \text { say . }
\end{aligned}
$$

Therefore $|c|_{i}^{(r+1)} \leqq E T^{r} P^{i}$, and

$$
\begin{aligned}
\left|x_{n}^{(0, r)}\right| & \leqq\left[\frac{\beta T^{r}}{P}+\frac{(1+P)}{P \alpha-1} E T^{r-1}\right] P^{n} \\
& =\left[\beta \frac{T}{P}+\frac{(1+P) E}{P \alpha-1}\right] T^{r-1} P^{n}=H T^{r-1} P^{n}, \text { say. }
\end{aligned}
$$


Observe that $\lim _{n \rightarrow \infty} c_{i}^{(n)}=0$. Let $X_{i}=\sum_{r=0}^{\infty} x_{i}^{(0, r)}$. The series converges absolutely, since

$$
\sum_{r=0}^{\infty}\left|x_{i}^{(0, r)}\right| \leqq H P^{i} \sum_{r=0}^{\infty} T^{r-1}, \quad T<1
$$

Define $Y_{\imath}^{(n)}$ by

$$
X_{i}=x_{i}^{(0,0)}+x_{i}^{(0,1)}+\cdots+x_{i}^{(0, n-1)}+Y_{i}^{(n)} .
$$

Then

$$
\left|Y_{i}^{(n)}\right| \leqq \sum_{r=n}^{\infty}\left|x_{i}^{(0, r)}\right| \leqq B P^{i} \sum_{r=n}^{\infty} T^{r-1}
$$

Therefore

$$
\lim _{n \rightarrow \infty} Y_{i}^{(n)}=0
$$

We have

$$
\sum_{j=i+1}^{\infty}\left|\lambda_{j}+b_{i j}\right|\left|x_{i}^{(0, r)}\right| \leqq N H T^{r-1} \sum_{j=i+1}^{\infty} P^{i}+H T^{r-1} \sum_{j=i+1}^{\infty}\left|\lambda_{j}\right| P^{i} .
$$

Both series on the right converge, and therefore $\sum_{j=i+1}^{\infty}\left(\lambda_{j}+b_{i j}\right) x_{j}^{(0, r)}$ converges absolutely for every $r$. Also

$$
\begin{aligned}
\sum_{j=i+1}^{\infty}\left|\lambda_{j}+b_{i j}\right|\left|Y_{j}^{(n)}\right| & \leqq N H\left(\sum_{r=n}^{\infty} T^{r-1}\right) \sum_{j=i+1}^{\infty} P^{i} \\
& +H\left(\sum_{r=n}^{\infty} T^{r-1}\right) \sum_{j=i+1}^{\infty}\left|\lambda_{i}\right| P^{i},
\end{aligned}
$$

and both series on the right converge. Therefore $\left.\sum_{j=t+1}^{\infty} \lambda_{j}+b_{i j}\right) Y_{j}^{(n)}$ converges absolutely. Moreover,

Therefore

$$
\lim _{n \rightarrow \infty} \sum_{j=i+1}^{\infty}\left(\lambda_{j}+b_{i j}\right) Y_{i}^{(n)}=0 .
$$

$$
\begin{aligned}
X_{i}+\sum_{j=i+1}^{\infty}\left(\lambda_{j}+b_{i j}\right) X_{j} \\
=x_{i}^{(0,0)}+\sum_{j=i+1}^{\infty}\left(\lambda_{j}+b_{i j}\right) x_{j}^{(0,0)} \\
\quad+x_{i}^{(0,1)}+\sum_{j=i+1}^{\infty}\left(\lambda_{j}+b_{i j}\right) x_{j}^{(0,1)} \\
\quad+\cdots+x_{i}^{(0, n-1)}+\sum_{j=i+1}^{\infty}\left(\lambda_{j}+b_{i j}\right) x_{i}^{(0, n-1)}
\end{aligned}
$$




$$
\begin{gathered}
+Y_{i}^{(n)}+\sum_{j=i+1}^{\infty}\left(\lambda_{j}+b_{i j}\right) Y_{i}^{(n)} \\
=\left(c_{i}-c_{i}^{(1)}\right)+\left(c_{i}^{(1)}-c_{i}^{(2)}\right)+\cdot \cdot \cdot+\left(c_{i}^{(n-1)}-c_{i}^{(n)}\right) \\
+Y_{i}^{(n)}+\sum_{j=i+1}^{\infty}\left(\lambda_{j}+b_{i j}\right) Y_{i}^{(n)} \\
=c_{i}+\left[-c_{i}^{(n)}+Y_{i}^{(n)}+\sum_{j=i+1}^{\infty}\left(\lambda_{j}+b_{i j}\right) Y_{j}^{(n)}\right] .
\end{gathered}
$$

As $n \rightarrow \infty$, the bracket approaches 0 .

Now the left hand member is independent of $n$. Therefore

i.e., $\left(X_{i}\right)$ is a solution of $(\mathrm{I})$.

$$
X_{i}+\sum_{j=i+1}^{\infty}\left(\lambda_{j}+b_{i j}\right) X_{j}=c_{i}
$$

Except for the condition $\left|x_{1}^{(0, r)}\right| \leqq \beta T^{r}, R<T<1$, the $x_{1}^{(0, r)}$ 's are arbitrary. Therefore $X_{1}$ is arbitrary. Consequently there are an infinity of solutions. We thus have

Theorem 3. If in the system

$$
x_{i}+\sum_{j=i+1}^{\infty}\left(\lambda_{j}+b_{i j}\right) x_{j}=c_{i} \quad(i=1,2,3, \cdot \cdot \cdot),
$$

the conditions $\left|c_{n}\right| \leqq M P^{n}, P<1$, and $\left|b_{i j}\right| \leqq N$ hold, then to every set $\left(\lambda_{i}\right)$ for which $\left|\lambda_{i}-1\right| \geqq \alpha>\left(N P^{2}+(N-1) P+1\right) / P(1-P)$ and $\sum_{j=1}^{\infty}\left|\lambda_{j}\right| P^{i}$ converges, there exist an infinity of solutions of $(\mathrm{I})$.

Corollary. From the inequality

$$
\left|X_{i}\right| \leqq H P^{i} \sum_{r=0}^{\infty} T^{r-1}
$$

we have the following result: An infinity of the solutions (for a given set $\lambda_{i}$ ) satisfy the inequality $\left|x_{i}\right| \leqq A P^{i}$, where $A$ is a constant (depending on $\alpha$ ).

CoRollary. If $\lambda_{j} \equiv \lambda$, then the system $x_{i}+\sum_{j=i+1}^{\infty}\left(\lambda+b_{i j}\right) x_{j}=c_{i}$ has an infinity of solutions for each $\lambda$ in $|\lambda-1|>\left(N P^{2}+(N-1) P+1\right) / P(1-P)$, if $\left|c_{i}\right| \leqq M P^{i}, P<1,\left|b_{i j}\right| \leqq N$, and an infinity of these solutions satisfy $\left|x_{i}\right| \leqq$ $A P^{i}, A$ depending on $\lambda$.

CoROllary. Consider the system $y_{i}+\sum_{j=i+1}^{\infty}\left(\lambda_{j}+b_{i j}\right) y_{j}=c_{i}$, where $\left|b_{i j}\right| \leqq$ $N ; P$ exists, $0<P<1$, such that $\sum_{j=1}^{\infty}\left|\lambda_{j}\right| P^{i}$ converges $;\left|\lambda_{i}-1\right| \geqq \alpha>$ $\left(N P^{2}+(N-1) P+1\right) / P(1-P)$. If this system has one solution, then it has an infinite number. 
For let $\bar{y}_{i}$ be a solution, and set $y_{i}=x_{i}+\bar{y}_{i}$. Then $x_{i}+\sum_{j=i+1}^{\infty}\left(\lambda_{j}+b_{i j}\right)=0$, which is a system satisfying the conditions of Theorem 3 . Therefore an infinity of solutions $x_{i}$, and consequently $y_{i}$, exist.

III. Solutions SATISfying the INEquality $\left|x_{i}\right| \leqq A P^{i *}$

In obtaining Theorem 3, we assumed that

$$
\left|x_{1}^{(0, r)}\right| \leqq \beta T^{r}, \quad R<T<1 .
$$

Let us now make a more particular choice; take

Then

$$
x_{1}^{(0, r)}=0 \quad(r=0,1,2, \cdots) .
$$

Therefore

$$
\begin{aligned}
M^{(r)} & =M R^{r}, \\
\mid c_{i}^{(r)} & \leqq M R^{r} P^{i}, \\
\left|x_{n}^{(0, r)}\right| & \leqq \frac{M(1+P)}{P \alpha-1} R^{r} P^{n} .
\end{aligned}
$$

$$
\left|X_{i}\right|=\left|\sum_{r=0}^{\infty} x_{i}^{(0, r)}\right| \leqq \frac{M(1+P)}{P \alpha-1}\left(\sum_{r=0}^{\infty} R^{r}\right) P^{i}=B M P^{i}
$$

where

$$
B=\frac{1+P}{(P \alpha-1)(1-R)} .
$$

We shall use these inequalities.

Consider again System (I) of Theorem 3. We found that for a given permissible set $\left(\lambda_{i}\right)$ an infinity of solutions exist satisfying

$$
\left|X_{i}\right| \leqq A P^{i} .
$$

We shall now show that every solution satisfying

$$
\left|X_{i}\right| \leqq A P^{i}
$$

$A$ a constant, is obtained by the method given in Theorem 3 . We shall however require a modification in the inequality for $\alpha$.

Let $X_{i}^{(0,0)}$ be a solution for which $\left|X_{i}^{(0,0)}\right| \leqq A P^{i}$. Then

$$
X_{i}^{(0,0)}+\sum_{j=i+1}^{\infty}\left(\lambda_{j}+b_{i j}\right) X_{i}^{(0,0)}=c_{i} .
$$

* The method used in this section is due to G. C. Evans, these Transactions, vol. 12 (1911), pp. 452-457. 
Consider the system

Define

$$
x_{i}+\sum_{j=i+1}^{\infty} \lambda_{j} x_{j}=c_{i}
$$

$$
x_{i}^{(1)}=x_{i}-X_{i}^{(0,0)} \text {. }
$$

Then $x_{i}^{(1)}+\sum_{j=i+1}^{\infty} \lambda_{j} x_{j}^{(1)}=c_{i}^{(1)}$, where $c^{(1)}=\sum_{j=i+1}^{\infty} b_{i j} X_{j}^{(0,0)}$. The series for $c_{i}^{(1)}$ obviously converges absolutely, and

$$
\left|c_{i}^{(1)}\right| \leqq A \frac{N P}{1-P} \cdot P^{i}, \quad=M^{(1)} P^{i}, \text { say. }
$$

Now let $X_{\imath}^{(0,1)}$ be a solution of

$$
x_{i}+\sum_{j=i+1}^{\infty}\left(\lambda_{j}+b_{i j}\right) x_{j}=c_{i}^{(1)}
$$

corresponding to the choice $x_{1}^{(0, r)}=0, r=0,1,2, \cdots$ in Theorem 3 . Then $\left|X_{i}^{(0,1)}\right| \leqq B M^{(1)} P^{i}$. Define $x_{i}^{(2)}=x_{i}^{(1)}-X_{i}^{(0,1)}$. Then $x_{i}^{(2)}+\sum_{j=i+1}^{\infty} \lambda_{j} x_{j}^{(2)}=c_{i}^{(2)}$, where $c_{i}^{(2)}=\sum_{j=1+1}^{\infty} b_{i j} X_{j}^{(0,1)}$. Then

$$
\left|c_{i}^{(2)}\right| \leqq \frac{N B M^{(1)} P}{1-P} \cdot P^{i}=B A\left(\frac{N P}{1-P}\right)^{2} P^{i}=M^{(2)} P^{i}
$$

In general,

where

$$
x_{i}^{(n)}+\sum_{j=i+1}^{\infty} \lambda_{j} x_{j}^{(n)}=c_{i}^{(n)}
$$

$$
c_{i}^{(n)}=\sum_{j=i+1}^{\infty} b_{i j} X_{j}^{(0, n-1)}, \quad x_{i}^{(n)}=x_{i}^{(n-1)}-X_{i}^{(0, n-1)},
$$

and $X^{(0, n-1)}$ is a solution of

$$
x_{i}+\sum_{j=i+1}^{\infty}\left(\lambda_{j}+b_{i j}\right) x_{j}=c_{i}^{(n-1)},
$$

corresponding to the choice $x_{1}^{(0, r)}=0, r=0,1,2, \cdots$; and

where

$$
\left|c_{i}^{(r)}\right| \leqq M^{(r)} P^{i}
$$

\section{Hence}

$$
M^{(r)}=\frac{N B P}{1-P} M^{(r-1)}, \quad M^{(1)}=\frac{N A P}{1-P} .
$$

$$
M^{(r)}=\frac{A}{B}-\left(\frac{B N P}{1-P}\right)^{r}=\frac{A}{B}-W^{r}
$$


where

$$
W=\frac{B N P}{1-P}
$$

Also,

$$
\left|X_{i}^{(0, r)}\right| \leqq B M^{(r)} P^{i}=A W^{r} P^{i}
$$

Assume $W<1$. This is equivalent to assuming that $R<\frac{1}{2}$; i.e., that

$$
\alpha>\frac{2 N P^{2}+(2 N-1) P+1}{P(1-P)} \text {. }
$$

Observe that this inequality implies the previous one,

$$
\alpha>\frac{N P^{2}+(N-1) P+1}{P(1-P)} \text {. }
$$

Since $W<1, \lim _{n \rightarrow \infty} c_{i}^{(n)}=0$.

Define $\bar{x}_{i}^{(0,0)}$ by the series

$$
\bar{x}_{i}^{(0,0)}=\sum_{r=0}^{\infty} X_{i}^{(0, r)} .
$$

The series converges absolutely, and

$$
\left|\bar{x}_{i}^{(0,0)}\right| \leqq A P^{i} \sum_{r=0}^{\infty} W^{r}=\frac{A}{1-W} P^{i} .
$$

Let

Then

$$
y_{i}^{(n)}=X_{i}^{(0, n+1)}+X_{i}^{(0, n+2)}+\cdots \cdot .
$$

$$
\left|y_{i}^{(n)}\right| \leqq A P^{i} \sum_{r=n+1}^{\infty} W^{r}
$$

therefore $\lim _{n \rightarrow \infty} y_{i}^{(n)}=0$. Again, from the inequality on $\left|X_{j}^{(0, r)}\right|$ we have

$$
\sum_{j=i+1}^{\infty}\left|\lambda_{j}\right|\left|X_{j}^{(0, r)}\right| \leqq A W^{r} \sum_{j=i+1}^{\infty}\left|\lambda_{i}\right| P^{j}
$$

which converges; hence

$$
\sum_{j=i+1}^{\infty}\left|\lambda_{j}\right|\left|y_{j}^{(n)}\right| \leqq A\left(\sum_{r=n+1}^{\infty} W^{r}\right) \sum_{j=i+1}^{\infty}\left|\lambda_{j}\right| P^{j} .
$$

Therefore $\lim _{n \rightarrow \infty} \sum_{j=i+1}^{\infty} \lambda_{j} y_{j}^{(n)}=0$. Then 


$$
\begin{aligned}
& \bar{x}_{i}^{(0,0)}+\sum_{j=i+1}^{\infty} \lambda_{j} \bar{x}_{j}^{(0,0)}=X_{i}^{(0,0)}+\sum_{j=i+1}^{\infty} \lambda_{j} X_{i}^{(0,0)}+\cdots \cdot+X_{i}^{(0 n)} \\
& \quad+\sum_{j=i+1}^{\infty} \lambda_{j} X_{j}^{(0, n)}+y_{i}^{(n)}+\sum_{j=i+1}^{\infty} \lambda_{j} y_{j}^{(n)} \\
& =\left(c_{i}-c_{i}^{(1)}\right)+\left(c_{i}^{(1)}-c_{i}^{(2)}\right)+\cdots+\left(c_{i}^{(n)}-c_{i}^{(n+1)}\right) \\
& \quad+y_{i}^{(n)}+\sum_{j=i+1}^{\infty} \lambda_{j} y_{j}^{(n)} \\
& =c_{i}+\left[-c_{i}^{(n+1)}+y_{i}^{(n)}+\sum_{j=i+1}^{\infty} \lambda_{j} y_{j}^{(n)}\right] .
\end{aligned}
$$

The bracket approaches zero as $n \rightarrow \infty$. Therefore $\bar{x}_{i}^{(0,0)}+\sum_{j=1+1}^{\infty} \lambda_{j} \bar{x}_{j}^{(0,0)}=$ $c_{i}$, i.e., $\left(\bar{x}_{i}^{(0,0)}\right)$ is a solution of $x_{i}+\sum_{j=i+1}^{\infty} \lambda_{j} x_{j}=c_{i}$.

Now we shall start with the solution $\bar{x}_{i}^{(0,0)}$ and show that one of the solutions $X_{i}$ of

$$
x_{i}+\sum_{j=i+1}^{\infty}\left(\lambda_{i}+b_{i j}\right) x_{j}=c_{i}
$$

resulting from the method of Theorem 3 is precisely $X_{1}^{(0,0)}$.

Define $\bar{x}_{i}^{(0,1)}, \bar{x}_{i}^{(0,2)}, \cdots$ as solutions of the systems

$$
\begin{aligned}
& \bar{x}_{i}^{(0,1)}+\sum_{j=i+1}^{\infty} \lambda_{j} \bar{x}_{j}^{(0,1)}=-\sum_{j=i+1}^{\infty} b_{i j} \bar{x}_{j}^{(0,0)}=e_{i}^{(1)}, \\
& \bar{x}_{i}^{(0,2)}+\sum_{j=i+1}^{\infty} \lambda_{j} \bar{x}_{j}^{(0,2)}=-\sum_{j=i+1}^{\infty} b_{i j} \bar{x}_{j}^{(0,1)}=e_{i}^{(2)},
\end{aligned}
$$

respectively. There are of course an infinite number of solutions of each of these systems, by Theorem 2. The precise solutions which we choose will be made evident later.

$X_{i}^{(0,0)}$ is a solution of $x_{i}+\sum_{j=i+1}^{\infty}\left(\lambda_{j}+b_{i j}\right) x_{j}=c_{i}$, and

$$
\left|X_{i}^{(0,0)}\right| \leqq A P^{i} \text {. }
$$

Let $X_{i}^{(1,0)}=-\sum_{r=1}^{\infty} X_{i}^{(0, r)}$; then $\left|X_{i}^{(1,0)}\right| \leqq A(W /(1-W)) P^{i}$.

Then $X_{i}^{(1,0)}$ satisfies $x_{i}+\sum_{j=i+1}^{\infty}\left(\lambda_{j}+b_{i j}\right) x_{j}=e_{i}^{(1)}$. For $X_{i}^{(1,0)}=X_{i}^{(0,0)}$ $-\bar{x}_{i}^{(0,0)}$, and

$$
X_{i}^{(0,0)}+\sum_{j=i+1}^{\infty}\left(\lambda_{j}+b_{i j}\right) X_{j}^{(0,0)}=c_{i}, \quad \bar{x}_{i}^{(0,0)}+\sum_{j=i+1}^{\infty} \lambda_{j} \bar{x}_{j}^{(0,0)}=c_{i} .
$$


We have

$$
\left|e_{i}^{(1)}\right| \leqq \frac{N A P}{(1-W)(1-P)} P^{i}
$$

Now define $X_{i}^{(1,1)}, X_{i}^{(1,2)}, \cdots, X_{i}^{(1, n)}, \ldots$ with respect to $X_{1}^{(1,0)}$ in the same way as $X_{1}^{(0,1)}, \cdots, X_{i}^{(0, n)}, \cdots$ were defined with respect to $X_{i}^{(0,0)}$. Then

$$
\left|X_{i}^{(1, r)}\right| \leqq A\left(\frac{W}{1-W}\right) W^{r} P^{i} \text {. }
$$

Choose $\bar{x}_{\imath}^{(0,1)}$ as that solution of

$$
x_{i}+\sum_{j=i+1}^{\infty} \lambda_{j} x_{j}=e_{i}^{(1)}
$$

corresponding to the set $X_{i}^{(1, r)}$ :

$$
\bar{x}_{i}^{(0,1)}=\sum_{r=0}^{\infty} X_{i}^{(1, r)}
$$

Now define $X_{\imath}^{(2,0)}=-\sum_{r=1}^{\infty} X_{\imath}^{(1, r)},=X_{i}^{(1,0)}-\bar{x}_{\imath}^{(0,1)}$. Then $X_{i}^{(2,0)}$ is a solution of

and

$$
x_{i}+\sum_{j=i+1}^{\infty}\left(\lambda_{j}+b_{i j}\right) x_{j}=e_{i}^{(2)}
$$

$$
\left|e_{i}^{(2)}\right| \leqq \frac{N A P}{(1-W)(1-P)}\left(\frac{W}{1-W}\right) P^{i} ; \quad\left|X_{i}^{(2,0)}\right| \leqq A\left(\frac{\Gamma W}{1-W}\right)^{2} P^{i}
$$

Define the corresponding set $X_{i}^{(2,1)}, X_{i}^{(2,2)}, \ldots$; then

$$
\left|X_{i}^{(2, r)}\right| \leqq A\left(\frac{W}{1-W}\right)^{2} W^{r} P^{i}
$$

Then choose $\bar{x}_{i}^{(0,2)}$ as that solution of

$$
x_{i}+\sum_{j=i+1}^{\infty} \lambda_{j} x_{j}=e_{i}^{(2)}
$$

which is determined by $X_{\imath}^{(2, r)}$ :

$$
\bar{x}_{i}^{(0,2)}=\sum_{r=0}^{\infty} X_{i}^{(2, r)} ;
$$

and so on. In general, we define

$$
X_{i}^{(k, 0)}=-\sum_{r=1}^{\infty} X_{i}^{(k-1, r)}, \quad=X_{i}^{(k-1,0)}-\bar{x}_{i}^{(0, k-1)} .
$$


Then $X_{i}^{(k, 0)}$ satisfies $x_{i}+\sum_{j=i+1}^{\infty}\left(\lambda_{j}+b_{i j}\right) x_{j}=e_{i}^{(k)}$, where $e_{i}^{(k)}=-\sum_{j=t+1}^{\infty}$ $b_{i j} \bar{x}_{j}^{(0, k-1)}$.

By induction we have $\left|X_{\imath}^{(\mathbf{k}, 0)}\right| \leqq A(W /(1-W))^{k} P^{i}$. We then define $X_{\mathfrak{\imath}}^{(\boldsymbol{k}, 1)}, X_{\mathfrak{\imath}}^{(\mathbf{k}, 2)}, \cdots$, and obtain $\left|X_{\mathfrak{\imath}}^{(\mathbf{k}, \tau)}\right| \leqq A(W /(1-W))^{\boldsymbol{k}} W^{r} P^{i}$. Also

$$
\left|e_{i}^{(k)}\right| \leqq \frac{N A P}{(1-P)(1-W)}\left(\frac{W}{1-W}\right)^{k-1} P^{i}
$$

Choose $\bar{x}_{i}^{(0, k)}$ as that solution of $x_{i}+\sum_{j=i+1}^{\infty} \lambda_{j} x_{i}=e_{i}^{(k)}$ which is given by $\bar{x}_{i}^{(0, k)}=\sum_{r=0}^{\infty} X_{i}^{(k, r)}$. Observe that

$$
\left|\bar{x}_{1}^{(0, k)}\right| \leqq \sum_{r=0}^{\infty} A\left(\frac{W}{1-W}\right)^{k} W^{r} P=\frac{A P}{1-W}\left(\frac{W}{1-W}\right)^{k} .
$$

This is in the form $\left|\bar{x}_{1}^{(0, k)}\right| \leqq \beta T^{\prime k}, T^{\prime}<1$, provided $W /(1-W)<1$; i.e., $W<\frac{1}{2}$, or $R<\frac{1}{3}$, which is equivalent to the inequality

$$
\alpha>\frac{3 N P^{2}+(3 N-1) P+1}{P(1-P)} \text {. }
$$

We assume this condition on $\alpha$. (It implies the previous condition $R<\frac{1}{2}$.) Consequently, by the method of Theorem 3,

$$
X_{i}=\sum_{r=0}^{\infty} \bar{x}_{i}^{(0, r)}
$$

is a solution of $x_{i}+\sum_{j=i+1}^{\infty}\left(\lambda_{j}+b_{i j}\right) x_{j}=c_{i}$.

It remains to identify $X_{i}$ with $X_{i}^{(0,0)}$. We see that

$$
\begin{aligned}
\bar{x}_{i}^{(0,0)}+ & \bar{x}_{i}^{(0,1)}+\cdots \cdot+\bar{x}_{i}^{(0, n)} \\
= & X_{i}^{(0,0)}+\left\{X_{i}^{(0,1)}+X_{i}^{(0,2)}+\cdot \cdot \cdot\right\} \\
& +X_{i}^{(1,0)}+\left\{X_{i}^{(1,1)}+X_{i}^{(1,2)}+\cdots \cdot\right\}+\cdot \cdot \\
& +X_{i}^{(n, 0)}+\left\{X_{i}^{(n, 1)}+\cdots \cdot\right\}
\end{aligned}
$$

where the right member, from the definition of $X_{t}^{(\mathbf{k}, 0)}, k=1,2, \cdots, n+1$, reduces to

$$
X_{i}^{(0,0)}-X_{i}^{(n+1,0)} .
$$

Now $\lim _{n \rightarrow \infty} X_{\imath}^{(n+1,0)}=0$. Therefore

$$
\lim _{n \rightarrow \infty}\left[\bar{x}_{i}^{(0,0)}+\cdots+\bar{x}_{i}^{(0, n)}\right]=X_{i}^{(0,0)} ;
$$


that is,

$$
X_{i}=X_{i}^{(0,0)}
$$

We have thus proved

TheOREM 4. If in the system

$$
x_{i}+\sum_{j=i+1}^{\infty}\left(\lambda_{i}+b_{i j}\right) x_{j}=c_{i} \quad(i=1,2, \cdot \cdot),
$$

the following inequalities hold:

$$
\begin{array}{r}
\left|c_{i}\right| \leqq M P^{i}, \quad P<1, \quad\left|b_{i j}\right| \leqq N ; \\
\left|\lambda_{i}-1\right| \geqq \alpha>\frac{3 N P^{2}+(3 N-1) P+1}{P(1-P)} ;
\end{array}
$$

and $\sum_{j=i+1}^{\infty}\left|\lambda_{i}\right| P^{j}$ converges, then every solution $x_{i}$ for which $\left|x_{i}\right| \leqq A P^{i}$ (of which there are an infinite number) is contained in the set obtained by the method of Theorem 3 , assigning to $x_{1}^{(0,0)}, x_{1}^{(0,1)}, \cdots$ suitable values in the range $\left|x_{1}^{(0, r)}\right| \leqq$ $\beta T^{r}, T<1$.

\section{THE POWER SERIES METHOD}

In Theorem 1 we made use of a Taylor series in $\lambda$, and found a solution by substituting the assumed series for $x_{i}$ into the system and equating coefficients. This method can be applied to other systems.

For example, consider the system

$$
x_{i}+\lambda \sum_{j=i+1}^{\infty} \alpha_{i j} x_{j}=c_{i} .
$$

Assume a solution $x_{i}=A_{0 i}+\cdots+A_{n i} \lambda^{n}+\cdots$. We find for the $A_{i j}$ 's the relations

$$
\begin{aligned}
& A_{0 i}=c_{i} \\
& A_{n i}=-\sum_{j=i+1}^{\infty} \alpha_{i j} A_{n-1, j}, \quad n>0 .
\end{aligned}
$$

It is readily shown that if $\left|c_{i}\right| \leqq C, \sum_{j=i+1}^{\infty}\left|\alpha_{i j}\right| \leqq P, i \geqq i_{0}$, then (I) has a bounded solution $\left(x_{i}\right)$ for every $\lambda$ in $|\lambda|<1 / P$. But this method does not appear to yield uniqueness properties. 
This method also gives the following theorem :

If

$$
\begin{aligned}
& \left|c_{i}\right| \leqq \alpha ; \quad \sum_{j=i+1}^{\infty}\left|\alpha_{i j}\right| \leqq S \quad(i=1,2, \cdot \cdot \cdot) \text {; } \\
& \varphi_{i j}(x) \text { is analytic }, \quad|x| \leqq M, \quad M>\alpha \\
& (i=1,2, \cdot \cdot \cdot j=i+1, i+2, \cdot \cdot \cdot) \text {; } \\
& \left|\varphi_{i j}(x)\right| \leqq N, \quad|x| \leqq M \text {; }
\end{aligned}
$$

then the system

$$
x_{i}+\lambda \sum_{j=i+1}^{\infty} \alpha_{i j} \varphi_{i j}\left(x_{j}\right)=c_{i}
$$

has a bounded solution for every $\lambda$ in $|\lambda| \leqq(M-\alpha) / 5 N S$.

It is an interesting fact that if we assume a power series solution in $1 / \lambda$, we obtain in many cases an infinite number of solutions. Let us consider again the system of Theorem 3 , where however we take $\lambda_{i} \equiv \lambda$ :

$$
x_{i}+\sum_{j=i+1}^{\infty}\left(\lambda+b_{i j}\right) x_{j}=c_{i}\left\{\begin{array}{l}
\left|c_{i}\right| \leqq M P^{i}, \quad P<1, \\
\left|b_{i j}\right| \leqq N .
\end{array}\right.
$$

Assume a solution

$$
x_{i}=A_{0 i}+\cdots+\frac{A_{n i}}{(\lambda-1)^{n}}+\cdots .
$$

On substituting in and equating coefficients we get

$$
\begin{array}{r}
\sum_{j=i+1}^{\infty} A_{0 j}=0, \quad A_{0 i}+\sum_{j=i+1}^{\infty}\left(b_{i j}+1\right) A_{0 j}+\sum_{j=i+1}^{\infty} A_{1 j}=c_{i}, \\
A_{n i}+\sum_{j=i+1}^{\infty}\left(b_{i j}+1\right) A_{n j}+\sum_{j=i+1}^{\infty} A_{n+1, j}=0, \quad n>0 .
\end{array}
$$

A solution of the first system is $A_{0 i}=0, i>1$. Observe that $A_{01}$ does not enter, and therefore can be taken arbitrarily. And in the general system, $A_{n 1}$ does not enter and is therefore arbitrary. This makes $x_{1}$ arbitrary, and we therefore obtain an infinity of solutions. Let us determine the $A_{i j}$ 's. We have the relation

$$
\sum_{j=i+1}^{\infty} A_{1 j}=c_{i}-A_{0 i}=c_{i}^{(1)}
$$

Therefore a solution is $A_{1, i+1}=c_{i}^{(1)}-c_{i+1}^{(1)}$. Also 


$$
\sum_{j=i+1}^{\infty} A_{2 j}=-A_{1 i}-\sum_{j=i+1}^{\infty}\left(b_{i j}+1\right) A_{1 j}=c_{i}^{(2)},
$$

and a solution is

$$
A_{2, i+1}=c_{i}^{(2)}-c_{i+1}^{(2)} \text {. }
$$

In general $A_{n, i+1}=c_{\imath}^{(n)}-c_{\imath+1}^{(n)}$, where $c_{\imath}^{(n)}=-A_{n-1, i}-\sum_{j=i+1}^{\infty}\left(b_{i j}+1\right) A_{n-1, j}$. By definition, $c_{i}^{(1)}=c_{i}-A_{0 i}$. Choose $A_{01}$ so that $\left|c_{1}-A_{01}\right| \leqq M P$; otherwise arbitrary. Then $\left|c_{i}^{(1)}\right| \leqq M P^{i}=M^{(1)} P^{i}$. Therefore $\left|A_{1, i+1}\right| \leqq M^{(1)}(1$ $+P) P^{i}$. Choose $A_{11}$ arbitrary except for the condition $\left|A_{11}\right| \leqq M(1$ $+P)$. Then

$$
\left|A_{1 i}\right| \leqq M(1+P) P^{i-1}=\frac{M(1+P)}{P}-P^{i}
$$

Therefore

$$
\left|c_{i}^{(2)}\right| \leqq \frac{M^{(1)}(1+P)}{P}-\left[1+\frac{(N+1) P}{1-P}\right] P^{i}=M^{(2)} P^{i} .
$$

Therefore $\left|A_{2, i+1}\right| \leqq M^{(2)}(1+P) P^{i}$. Choose $\left|A_{2,1}\right| \leqq M^{(2)}(1+P)$; then $\left|A_{2 i}\right| \leqq\left(M^{(2)}(1+P) / P\right) P^{i} ;$ and so on. We obtain, finally, $\left|c_{i}^{(n)}\right| \leqq M^{(n)} P^{i}$, where

Therefore

$$
\begin{aligned}
M^{(n)} & =R M^{(n-1)}=\cdot \cdot=M R^{n-1}, \\
R & =\left(1+\frac{P(N+1)}{1-P}\right)\left(\frac{1+P}{P}\right) .
\end{aligned}
$$

$$
\begin{aligned}
& \left|c_{i}^{(n)}\right| \leqq M R^{n-1} P^{i} \\
& \left|A_{n i}\right| \leqq \frac{M(1+P)}{P}-R^{n-1} P^{i}
\end{aligned}
$$

on choosing

$$
\left|A_{n 1}\right| \leqq M R^{n-1}(1+P)
$$

Therefore

$$
\left|x_{i}\right| \leqq P^{i} \frac{M(1+P)}{R P}-\sum_{n=0}^{\infty} \frac{R^{n}}{|\lambda-1|^{n}}=T_{i}
$$

which converges if $|\lambda-1|>R$; i.e., if

$$
|\lambda-1|>\frac{(1+P)(N P+1)}{P(1-P)} \text {. }
$$

Also, $T_{i}+\sum_{j=i+1}^{\infty}\left|\lambda+b_{i j}\right| T_{j}$ converges. Hence we can sum by columns. We thus have the following theorem: 
If $\left|c_{i}\right| \leqq M P^{i}, P<1,\left|b_{i j}\right| \leqq N$, and $|\lambda-1|>(1+P)(N P+1) / P(1-P)$, then the system $x_{i}+\sum_{j=i+1}^{\infty}\left(\lambda+b_{i j}\right) x_{j}=c_{i}$ has an infinity of solutions.

So we obtain some of the results of Theorem 3. But the power series method does not extend to the case where the $\lambda_{j}$ 's are not all equal; and even in the case just treated, the $\lambda$-region for which the proof is valid is not as extended as the region found by the method of Theorem 3 .

Rice INSTITUTE,

Houston, Texas. 